\title{
Finite-time synchronization of tunnel-diode-based chaotic oscillators
}

\author{
Patrick Louodop, ${ }^{1}$ Hilaire Fotsin, ${ }^{1}$ Michaux Kountchou, ${ }^{1}$ Elie B. Megam Ngouonkadi, ${ }^{1}$ \\ Hilda A. Cerdeira, ${ }^{2}$ and Samuel Bowong ${ }^{3}$ \\ ${ }^{1}$ Laboratory of Electronics and Signal Processing Faculty of Science, Department of Physics, University of Dschang, \\ P.O. Box 67, Dschang, Cameroon \\ ${ }^{2}$ Instituto de Física Teórica, UNESP, Universidade Estadual Paulista, Rua Dr. Bento Teobaldo Ferraz 271, \\ Bloco II, Barra Funda, 01140-070 São Paulo, Brazil \\ ${ }^{3}$ Laboratory of Applied Mathematics, Department of Mathematics and Computer Science, Faculty of Science, \\ University of Douala, P.O. Box 24157, Douala, Cameroon
}

(Received 3 June 2013; published 26 March 2014)

\begin{abstract}
This paper addresses the problem of finite-time synchronization of tunnel diode based chaotic oscillators. After a brief investigation of its chaotic dynamics, we propose an active adaptive feedback coupling which accomplishes the synchronization of tunnel-diode-based chaotic systems with and without the presence of delay(s), basing ourselves on Lyapunov and on Krasovskii-Lyapunov stability theories. This feedback coupling could be applied to many other chaotic systems. A finite horizon can be arbitrarily established by ensuring that chaos synchronization is achieved at a pre-established time. An advantage of the proposed feedback coupling is that it is simple and easy to implement. Both mathematical investigations and numerical simulations followed by PSPICE experiment are presented to show the feasibility of the proposed method.
\end{abstract}

DOI: 10.1103/PhysRevE.89.032921

PACS number(s): 05.45.Xt, 05.45.Gg

\section{INTRODUCTION}

Since chaotic systems were discovered, considerable interest arose in developing and analyzing various systems that exhibit chaos due to their importance in many fields of sciences [1-3]. In biology, epidemiology, or climatology, investigating mathematical models is a part of the strategies used to better comprehend the phenomenon [1,2,4-8]. However, there exist difficulties to understand some of them because of the lack of data or the time taken to produce reliable data. For example, in epidemiology, some deceases have to be observed for a long period before they can be modeled [4]. To turn around these problems, electrical circuits (analog computers) are built to allow the observation of the effect of different parameters. Nevertheless, these electrical circuits have to be synchronized with the time history of data as much as possible to be reliable. Hence, synchronization becomes an important property to be studied.

Another characteristic of synchronization is its applications in chaos based cryptography [9-11]. Even if this problem can be found in a number of papers, most of them deal with infinite settling time [9-11]. If we consider, for example, the application of synchronization in secure communications, the range of time during which the chaotic oscillators are not synchronized corresponds to the range of time during which the encoded message can unfortunately not be recovered or sent. More than a difficulty, this is a catastrophe in digital telecommunications, since the first bits of standardized bit strings always contain signalization data, i.e., the "identity card" of the message. Hence, it clearly appears that the synchronization time has to be known and minimized, so that the chaotic oscillators synchronize as fast as possible. In this context, it is fair to say that there is a need to study finite-time chaos synchronization. This characteristic is also helpful because of its advantages in its applications in engineering. Hence, it is important to investigate the finite-time stability of nonlinear systems [12-15].
Furthermore, even if delay complicates synchronization, dynamical systems with delay(s) are abundant in nature. They occur in a wide variety of physical, chemical, engineering, economics, and biological systems and their networks. There are many examples where delay plays an important role. Some of these examples are listed and presented by M. Lakshmanan and D. V. Senthilkumar in [16]. The mathematical description of delay dynamical systems will naturally involve the delay parameter in some specified way. This can be in the form of differential equations with delay or difference equations with delay and so on [16]. Delay differential equations are given in many ways. Again, we refer the reader to [16] for more information.

In telecommunications, the delay notion is inescapable, for example, because of the relative distance between the transmitter and the receiver. Thus, the use of fast signals is indicated. A solution is provided by electronic components such as tunnel diodes. Developed in 1956 by Léo Esaki, the tunnel diode is a nonlinear device used in very high frequency amplifiers with low noise and in microwave conception. The tunnel diode based sources can provide rf signals above $500 \mathrm{GHz}$. Fundamental oscillations at $712 \mathrm{GHz}$ from a resonant tunnel diode oscillator were demonstrated by Brown et al. in 1991 [17]. Recently, fundamental frequency oscillations of a resonant tunnel diode oscillator close to 831 and $915 \mathrm{GHz}$ and $1.04 \mathrm{THz}$ at room temperature were reported by Suzuki et al. [18] - more details can be found in the Ph.D. thesis of L. Wang (2011) of the University of Glasgow [19], and references therein. Considering the frequency capability of the tunnel diode, it can be used as an efficient source for chaotic signals in wireless communications; in particular, H.-P. Ren et al. [20] show that, for some particular chaotic signals, the Lyapunov exponents remain unaltered through multipath propagation, thus making these systems able to sustain communications with chaotic signals. However, experimental observations of generalized synchronization phenomena in microwave oscillators have been done by B. S. Dmitriev 
et al. [21], and their application to secure communications has been carried out by A. A. Koronovskii et al. [22]. Nevertheless, the synchronization schemes used in [20-22] are dealing with asymptotical synchronization and can only guarantee that two systems achieve synchronization as time tends to infinity, while in real-world applications one always expects that two systems achieve synchronization within a finite and/or predetermined time. Besides, finite-time synchronization has been confirmed to have better robustness against parametric and external disturbances than the asymptotical one [23,24]-thus, the importance of studying finite-time synchronization of chaotic systems. The use of tunnel diodes to construct chaotic systems was also reported by A. Pikovskii [25], A. Fradkov [26], and others. For the purpose of this work, we base ourselves on the circuit proposed by A. Y. Markov et al. [26], where we replace the used tunnel diode by the one with reference number 1N3858, for which the PSPICE model is available on the Internet.

In this paper we study an active adaptive finite-time synchronization of tunnel diodes based chaotic oscillators with (the first case) and without (the second and third cases) time delay in both the drive and response systems. Therefore, we will investigate the adaptive synchronization of chaotic systems using just one master state variable as output to construct the nonlinear feedback coupling. A robust adaptive response system will be therefore designed to always globally synchronize the given driven tunnel diode based oscillator. These results are of significant interest to infer relationships between nonlinear feedback coupling, time delay, and finitetime synchronization. To the best of our knowledge, this active adaptive finite-time synchronization of chaotic systems using a nonlinear feedback coupling has not yet been reported in the literature.

The manuscript is organized as follows: In Sec. II, the circuit model and its chaotic dynamics are investigated. Section III deals with the adaptive synchronization problem. In this section the active controller is designed and the theoretical settling time in the case of systems without delay is developed. Numerical simulations are performed to show the effectiveness of the synchronization scheme. In Sec. IV, we investigate the cases of delayed systems, considering two cases. The theoretical settling is also obtained and the numerical results are presented graphically to prove the effectiveness of the scheme. In the last section we present the conclusions.

\section{THE MODEL AND ITS PROPERTIES}

Before starting to analyze adaptive synchronization and delay, we introduce the elements of our circuit. Its electronic diagram is given in Fig. 1(a), where the tunnel diode model is the $1 \mathrm{~N} 3858$ with the current-voltage characteristic given in Fig. 1(b). The chaotic circuit is a $R L C$ oscillator, where $E$ is a continuous voltage source.

The circuit diagram presents two capacitors $C_{1}$ and $C_{2}$ and an inductor $L$. The nonlinearity is introduced by the tunnel diode, which has its current-voltage characteristic depicted in Fig. 1(b). As an approximation of this current-voltage characteristic, we use the following relation [26]:

$$
i\left(V_{1}\right)=a_{1}\left(V_{1}-b\right)^{3}-a_{2}\left(V_{1}-b\right)+a_{3},
$$
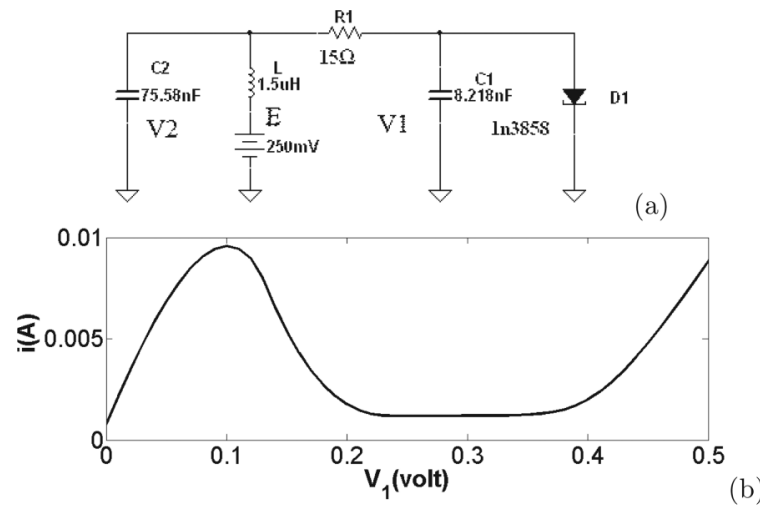

FIG. 1. (a) Circuit diagram and (b) current voltage characteristic of tunnel diode $1 \mathrm{~N} 3858$.

where $a_{1}, a_{2}$, and $a_{3}$ are positive constants. The circuit can be described by the following system of differential equations:

$$
\begin{aligned}
& \dot{x}_{1}(\tau)=\alpha\left[x_{2}-x_{1}-r f\left(x_{1}\right)\right] \\
& \dot{x}_{2}(\tau)=\beta\left(x_{1}-x_{2}+r x_{3}\right) \\
& \dot{x}_{3}(\tau)=\gamma\left(E-x_{2}\right),
\end{aligned}
$$

where

$$
\begin{gathered}
c=\frac{C_{2}}{C_{1}}, \quad r=R_{1}, \quad q=\frac{r}{L w}, \quad \tau=w t, \\
\varrho=1 V, \quad I=1 A, \quad x_{1}=\frac{V_{1}}{\varrho}, \quad x_{2}=\frac{V_{2}}{\varrho}, \\
x_{3}=\frac{i}{I}, \quad \alpha=\frac{c}{q}, \quad \beta=\frac{1}{q}, \quad \gamma=\frac{q}{r}, \quad \text { and } \\
f\left(x_{1}\right)=a_{1}\left(x_{1}-b\right)^{3}-a_{2}\left(x_{1}-b\right)+a_{3},
\end{gathered}
$$

where $V_{1}$ and $V_{2}$ are the voltages at landmarks of $C_{1}$ and $C_{2}$, respectively; $i$ is the current which flows through the inductor; and $w$ is a constant. Note that Eq. (2) is similar to the so-called modified Chua's circuit [27].

Before entering into the description of the system under study we present the chaotic behavior of the circuit due to the influence of parameter $\alpha$ on the evolution of the one-dimensional Lyapunov exponent [Fig. 2(a)] and on the bifurcation diagram [Fig. 2(b)]. These two graphs allow us to determine the value of the control parameter, leading to a chaotic behavior of the system through period doubling. Figure 2(a) shows that for some values of the parameter $\alpha$ the system Eq. (2) has a positive Lyapunov exponent.

Figures 3(a) and 3(b) show the graphs $x_{2}$ vs $x_{1}$ and $x_{1}$ vs $x_{3}$ obtained from PSPICE simulations of the circuit of Fig. 1(a). An approximate behavior is displayed with the parameters $c=8.4, q=3.35, E=0.25, r=16, a_{1}=$ $1.3242872, a_{2}=0.06922314, a_{3}=0.005388$, and $b=0.167$ and initial conditions $x_{1}(0)=0.15, x_{2}(0)=0.27$, and $x_{3}(0)=$ 0.008 .

In Fig. 4 the space $(c, q)$ for which the system behaves chaotically is shown in black. With $\alpha=2.507462687$ and the values of the parameters given above, basing ourselves on Fig. 4, it follows that the Lyapunov exponent is positive and the system shows chaotic behavior (Fig. 5). 

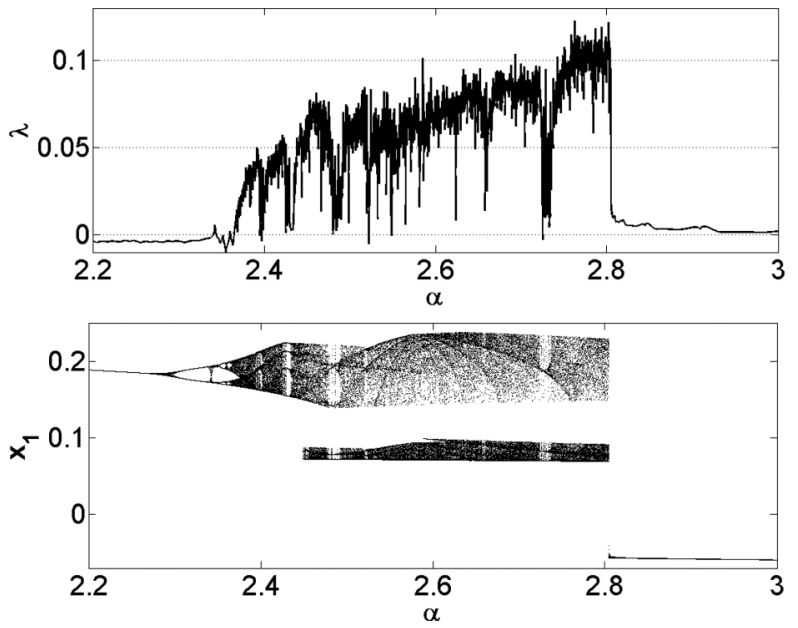

(a)

FIG. 2. (a) One-dimensional Lyapunov exponent and (b) bifurcation diagram when $E=0.253208$ and $q=3.6$.

The graphs of Fig. 6 show the Poincaré sections which help to prove the chaotic behavior of the model [Eq. (2)].

\section{ADAPTIVE SYNCHRONIZATION}

The starting point of the control law design is always a mathematical model of the real system which describes the system response to various control inputs. It is usually assumed that such a model is known rather completely. However, in practice the model describes the real system with some degree of uncertainty. To cope with this uncertainty in such cases, methods of the adaptive control theory can be employed. The concept of chaos synchronization emerged much later, not until the gradual realization of the usefulness of chaos by scientists and engineers. Chaotic signals are usually broadband and noiselike. Because of these properties, synchronized

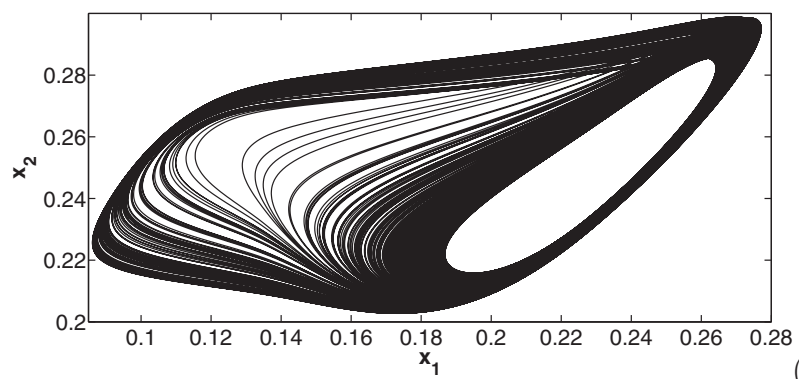

(a)

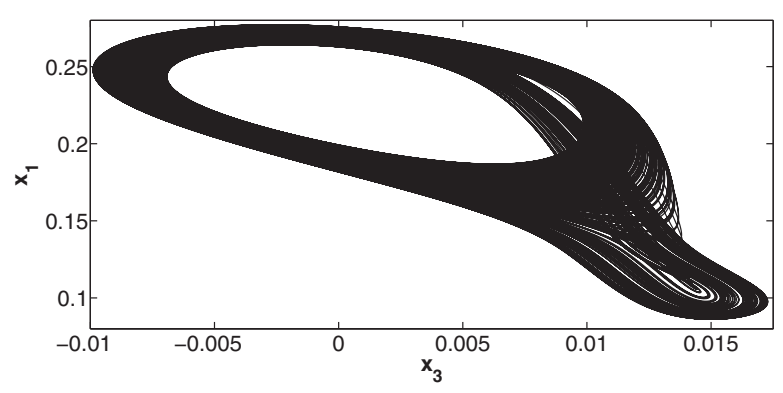

FIG. 3. Chaotic attractor obtained through PSPICE simulation of the circuit of Fig. 1(a).

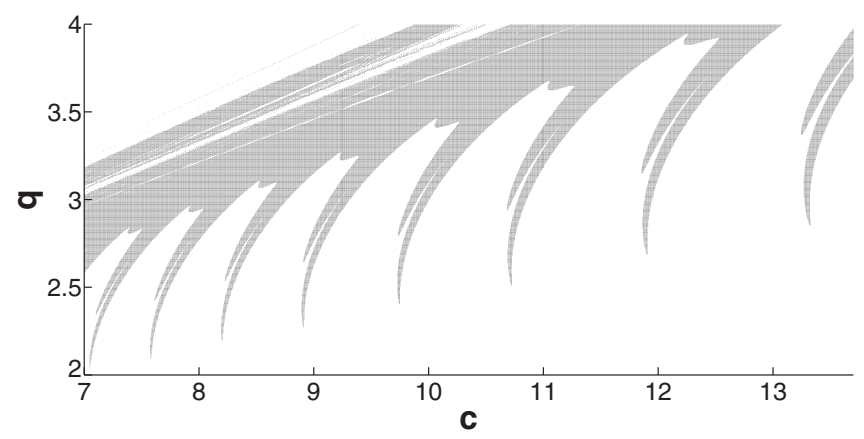

FIG. 4. Pair $(c, q)$ for which chaos occurs with $E=0.25$.

chaotic systems can be used as cipher generators for secure communication.

In the following section, we investigate two identical systems of the type described in the previous section, where the system drive $x_{i}(\tau), i=1,2,3$ drives the response system $y_{i}(\tau), i=1,2,3$.

\section{A. Design of the nonlinear controller}

Here we investigate the finite-time synchronization of tunnel diode based chaotic oscillators described by Eq. (2). For this purpose we consider the corresponding response system described by

$$
\begin{aligned}
& \dot{y}_{1}=\alpha\left[y_{2}-y_{1}-r f\left(y_{1}\right)\right]-\zeta k \operatorname{sign}\left(y_{1}-x_{1}\right)-\zeta u(\tau) \\
& \dot{y}_{2}=\beta\left[y_{1}-y_{2}+r y_{3}-2\left(y_{1}-x_{1}\right)\right] \\
& \dot{y}_{3}=\gamma\left(E-y_{2}\right)
\end{aligned}
$$

where $k$ and $\zeta$ are positive constant gains to be defined by the designer. The term $-2\left(y_{1}-x_{1}\right)$ added in the

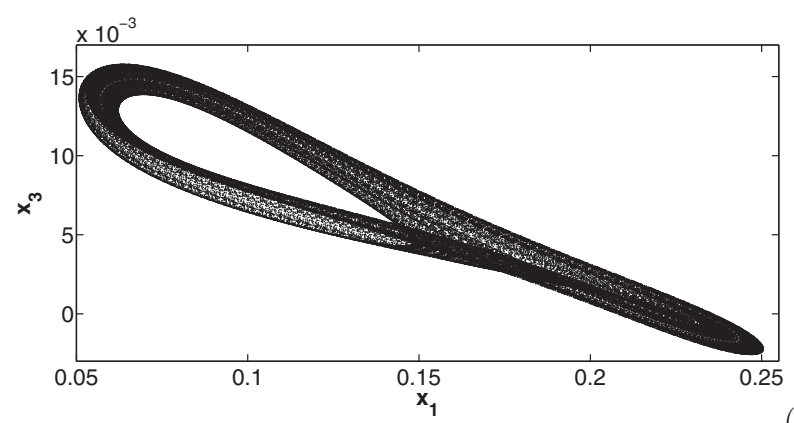

(a)

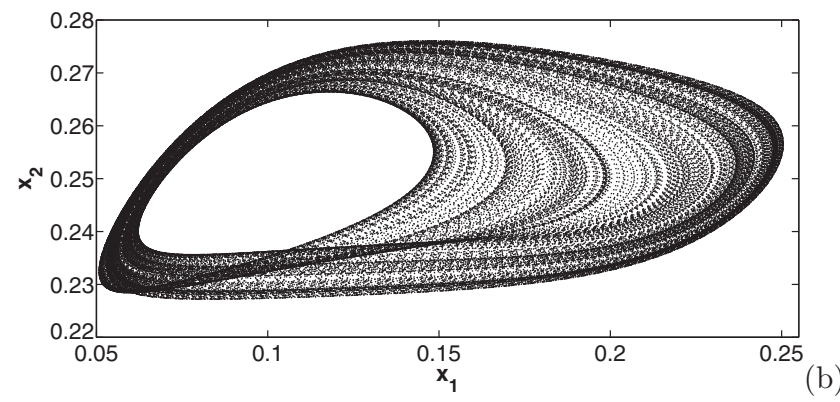

FIG. 5. Chaotic attractor obtained through numerical simulation of Eq. (2). 

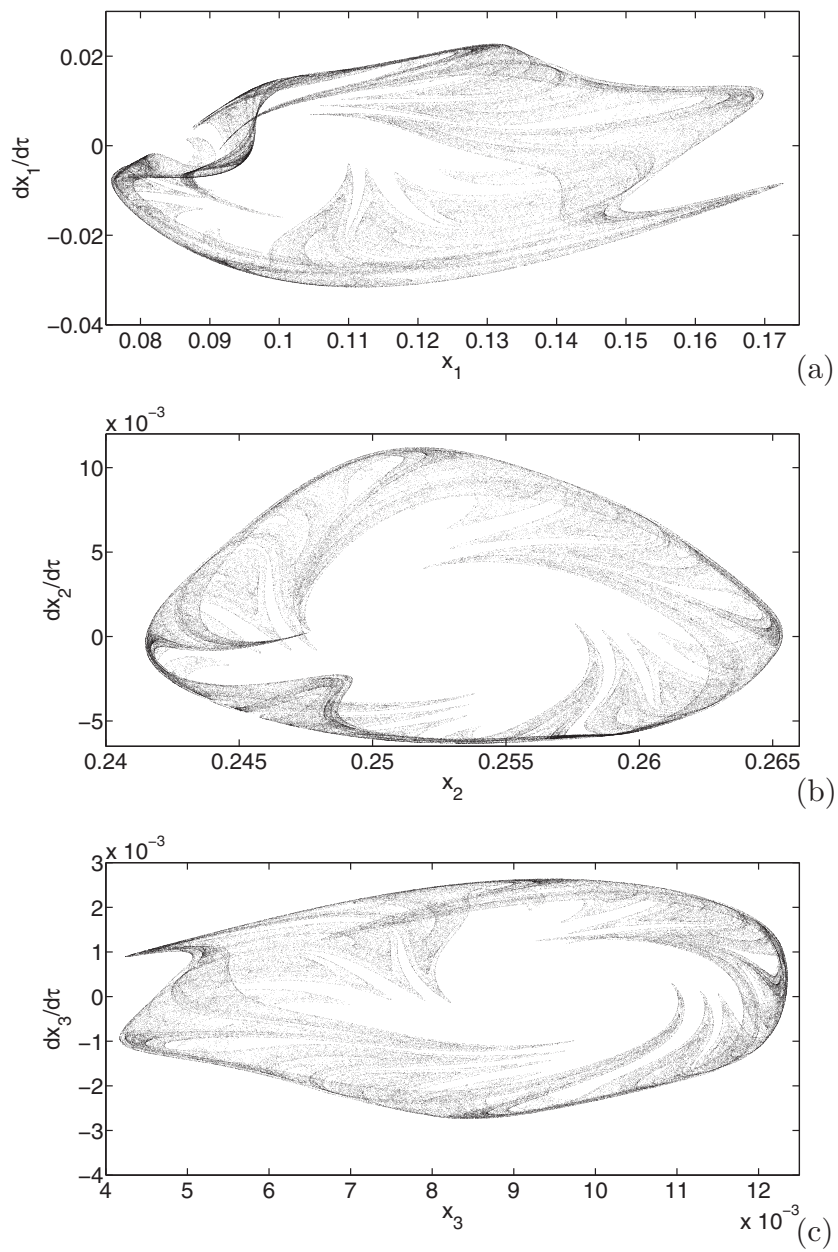

FIG. 6. Poincaré sections with $E=0.253205, c=8.846$, and $q=3.6$.

$y_{2}$ axis stabilizes the slave system. The nonlinear controller is expressed as $-\zeta k \operatorname{sign}\left(y_{1}-x_{1}\right)-\zeta u(\tau)$, where $u(\tau)$ is the adaptive feedback coupling designed to achieve finite-time synchronization. All parts of the used controller contribute to synchronize both drive-response systems at an established time and also to stabilize the response dynamics.

Let us define the synchronization error as follows:

$$
e_{i}(\tau)=y_{i}(\tau)-x_{i}(\tau), \quad \text { with } i=1,2,3 .
$$

Subtracting Eq. (2) from Eq. (3) and using Eq. (4), we obtain

$$
\begin{aligned}
& \dot{e}_{1}=\alpha\left[e_{2}-e_{1}-\phi_{0}\left(x_{1}, y_{1}\right)\right]-\zeta k \operatorname{sign}\left(e_{1}\right)-\zeta u(\tau) \\
& \dot{e}_{2}=\beta\left[-e_{1}-e_{2}+r e_{3}\right] \\
& \dot{e}_{3}=-\gamma e_{2},
\end{aligned}
$$

where $\phi_{0}\left(x_{1}, y_{1}\right)=r\left[f\left(y_{1}\right)-f\left(x_{1}\right)\right]$.

The synchronization problem can be stated as follows: considering the transmitter Eq. (2) and the receiver Eq. (3) with any initial conditions, $e_{1}(0)=y_{1}(0)-x_{1}(0), e_{2}(0)=$ $y_{2}(0)-x_{2}(0), e_{3}(0)=y_{3}(0)-x_{3}(0)$, it is our aim to design the form of the function $u(\tau)$ which synchronizes the orbits of both the transmitter and the response systems and thus provides the stabilization of Eq. (5) at an established finite-time $\tau_{s}$, i.e.,

$$
\begin{aligned}
& \lim _{t \rightarrow \tau_{s}} x_{i}(\tau)=y_{i}(\tau), \quad i=1,2,3, \\
& \lim _{\tau \rightarrow \tau_{s}}\|e(\tau)\|=0 .
\end{aligned}
$$

where $\tau_{s}$ is the settling time. We consider that the function $\phi_{0}\left(x_{1}, y_{1}\right)$ respects the Lipschitz condition; i.e., there exists a positive constant $\chi_{0}$ such that $\left|\phi_{0}\left(x_{1}, y_{1}\right)\right| \leqslant \chi_{0}\left|e_{1}(\tau)\right|$.

\section{B. Main results}

The global finite-time synchronization is achieved when the Lyapunov function candidate is proper [15], namely, the time derivative of the used Lyapunov function is bounded by a negative constant.

Let us consider the following candidate for the Lyapunov function [28]:

$$
V=\frac{1}{2}\left(\frac{e_{1}^{2}}{\alpha}+\frac{e_{2}^{2}}{\beta}+\frac{r e_{3}^{2}}{\gamma}\right)+|u(\tau)| .
$$

The time derivative along the trajectories of the system of Eqs. (5) yields

$$
\begin{aligned}
\dot{V}= & -e_{1}^{2}-e_{2}^{2}-\phi_{0}\left(x_{1}, y_{1}\right) e_{1}-\frac{\zeta k}{\alpha}\left|e_{1}\right|-\frac{\zeta u(\tau)}{\alpha} e_{1} \\
& +\operatorname{sign}(u) \dot{u}(\tau), \\
\dot{V} \leqslant & -e_{1}^{2}-e_{2}^{2}+\left|\phi_{0}\left(x_{1}, y_{1}\right)\right|\left|e_{1}\right|-\frac{\zeta k}{\alpha}\left|e_{1}\right|-\frac{\zeta u(\tau)}{\alpha} e_{1} \\
& +\operatorname{sign}(u) \dot{u}(\tau), \\
\dot{V} \leqslant & -\left(1-\chi_{0}\right) e_{1}^{2}-e_{2}^{2}-\frac{\zeta k}{\alpha}\left|e_{1}\right|-\frac{\zeta u(\tau)}{\alpha} e_{1} \\
& +\operatorname{sign}(u) \dot{u}(\tau) .
\end{aligned}
$$

Letting $\chi_{0}<1$ and $\zeta=\alpha$,

$$
\dot{V} \leqslant-k\left|e_{1}\right|-u(\tau) e_{1}+\operatorname{sign}(u) \dot{u}(\tau) .
$$

Considering the finite-time stability theory applied in [15], it follows that there exists a certain positive constant $p$ such that the following relation holds:

$$
\dot{V} \leqslant-p .
$$

If the objective is reached, the theoretical finite time for synchronization is obtained by integrating Eq. (10) from zero to $\tau_{s}$. Thus, one has

$$
\tau_{s} \leqslant \frac{1}{2 p}\left(\frac{e_{1}^{2}(0)}{\alpha}+\frac{e_{2}^{2}(0)}{\beta}+\frac{r e_{3}^{2}(0)}{\gamma}\right)+\frac{|u(0)|}{p} .
$$

Therefore, in accordance with Eq. (10), the controller $u(\tau)$ is designed through the following relation:

$$
\dot{u}=\operatorname{sign}(u)\left(k\left|e_{1}\right|+u e_{1}-p\right),
$$

and we construct the slave system Eq. (3) as follows:

$$
\begin{aligned}
\dot{y}_{1} & =\alpha\left[y_{2}-y_{1}-r f\left(y_{1}\right)\right]-\zeta k \operatorname{sign}\left(y_{1}-x_{1}\right)-\zeta u \\
\dot{y}_{2} & =\beta\left[y_{1}-y_{2}-r y_{3}-2\left(y_{1}-x_{1}\right)\right] \\
\dot{y}_{3} & =\gamma\left[E-y_{2}\right] \\
\dot{u} & =\operatorname{sign}(u)\left(k\left|e_{1}\right|+u e_{1}-p\right) .
\end{aligned}
$$



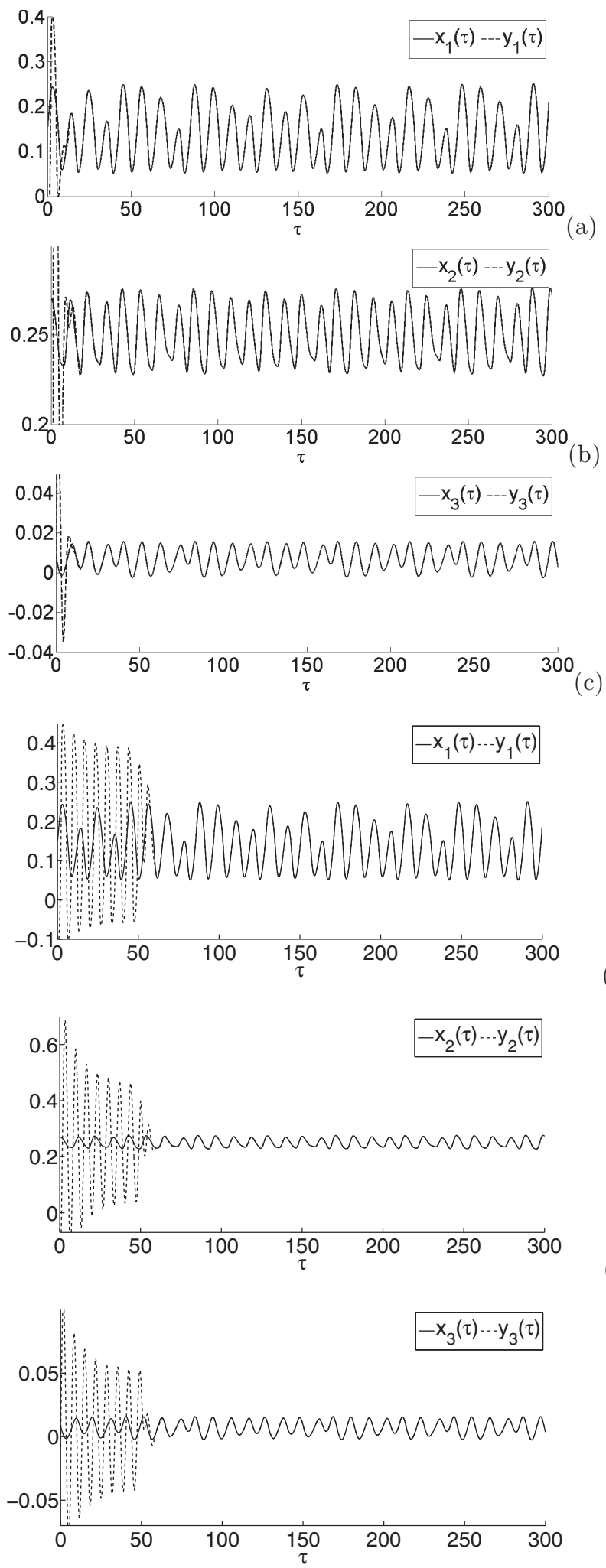

FIG. 7. Time histories of state variables: the solid line depicts the drive system, and the dashed line depicts the response system.

\section{Numerical results}

To illustrate the effectiveness of the proposed scheme we present some numerical results for the initial conditions given by $\left(x_{1}(0), x_{2}(0), x_{3}(0)\right)=(0.15,0.27,0.008)$ and
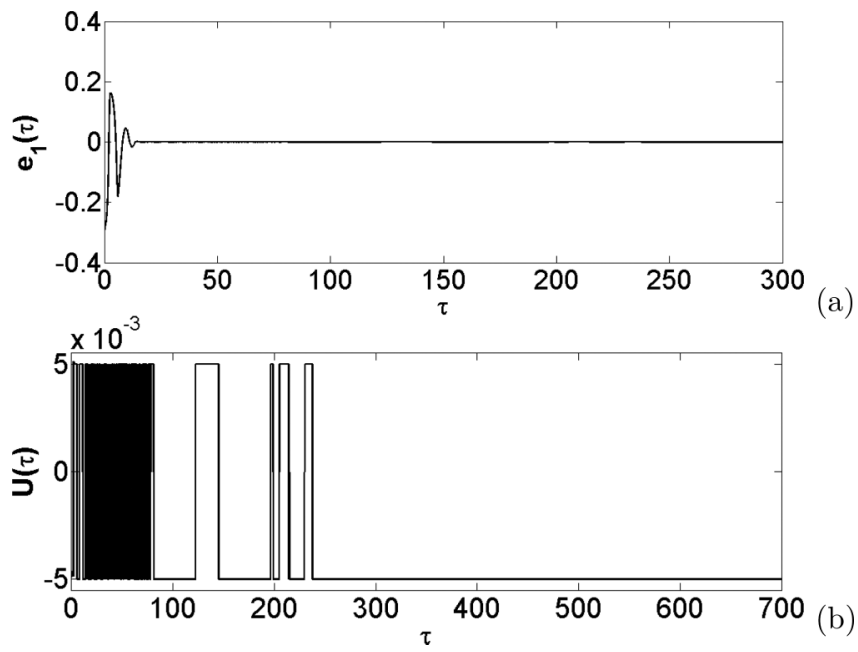

FIG. 8. Time histories of (a) $e_{1}(\tau)$ and (b) the controller $u(\tau)$.

$\left(y_{1}(0), y_{2}(0), y_{3}(0)\right)=(-0.15,-0.27,-0.008)$; the parameters $p=0.001, k=0.005$; and the initial condition of controller $u(0)=0.0001$. The integration time step was taken as $10^{-4}$. For such values, the theoretical settling time is determined to be $\tau_{s \mathrm{TH}}=521.0579$. The graphs of Figs. 7(a)-7(c) show the time dependence of the master system state variables (solid lines) and the corresponding slave system state variables (dashed lines), while those in Figs. 7(d)-7(f) show the same graphs for another set of initial conditions, for which the controller was turned on at $\tau=50$ to show the dynamics before and after synchronization. From the graphs in Fig. 8 we confirm that the synchronization is reached in finite time. It is observed that synchronization is reached at time $\tau_{s \mathrm{NU}} \simeq$ 245.3, which respects the finite-time condition $\tau_{s \mathrm{NU}} \leqslant \tau_{s \mathrm{TH}}$ [Fig. 8(b)].

The value of the finite time of stability depends strongly on the values of parameters $p$. Considering $k \simeq 0.005$ the following graphs represent the behavior of the error state $e_{1}(\tau)$ and of the adaptive parameter $u(\tau)$ for two given values of $p$ [Fig. 9(a)]. In principle $p$ can take any values, but to deal with finite-time stability it should be small. The graphs in Fig. 9 represent the behavior of both $e_{1}(\tau)$ and $u(\tau)$ for $p=$ 0.0017 and 0.002 [Figs. 9(a) and 9(b) and Figs. 9(c) and 9(d), respectively). In this paper we define the numerical finite time of convergence as the end time of the transitory phase of the time evolution of $e_{1}(\tau)$. For the graphs in Figs. 9(a) and 9(b) the numerical finite time is $\tau_{s \mathrm{NU}} \simeq 100$ and the theoretical finite time is $\tau_{s \mathrm{TH}} \simeq 306.5047$, while for the ones in Figs. 9(c) and $9(\mathrm{~d})$ the theoretical finite time is $\tau_{s \mathrm{TH}} \simeq 260.5290$ and the numerical finite time is $\tau_{s \mathrm{NU}}>\tau_{s \mathrm{TH}}$. Thus we see that the finite-time condition $\tau_{s \mathrm{NU}} \leqslant \tau_{s \mathrm{TH}}$ is fulfilled in both cases.

\section{FINITE-TIME TIME-DELAY SYNCHRONIZATION OF TUNNEL DIODE BASED OSCILLATORS}

\section{A. Systems with internal delay}

\section{Synchronization analysis}

In this section, we investigate the finite-time synchronization of delayed tunnel-diode-based chaotic oscillators. We first consider the presence of one delay affecting the nonlinearity 

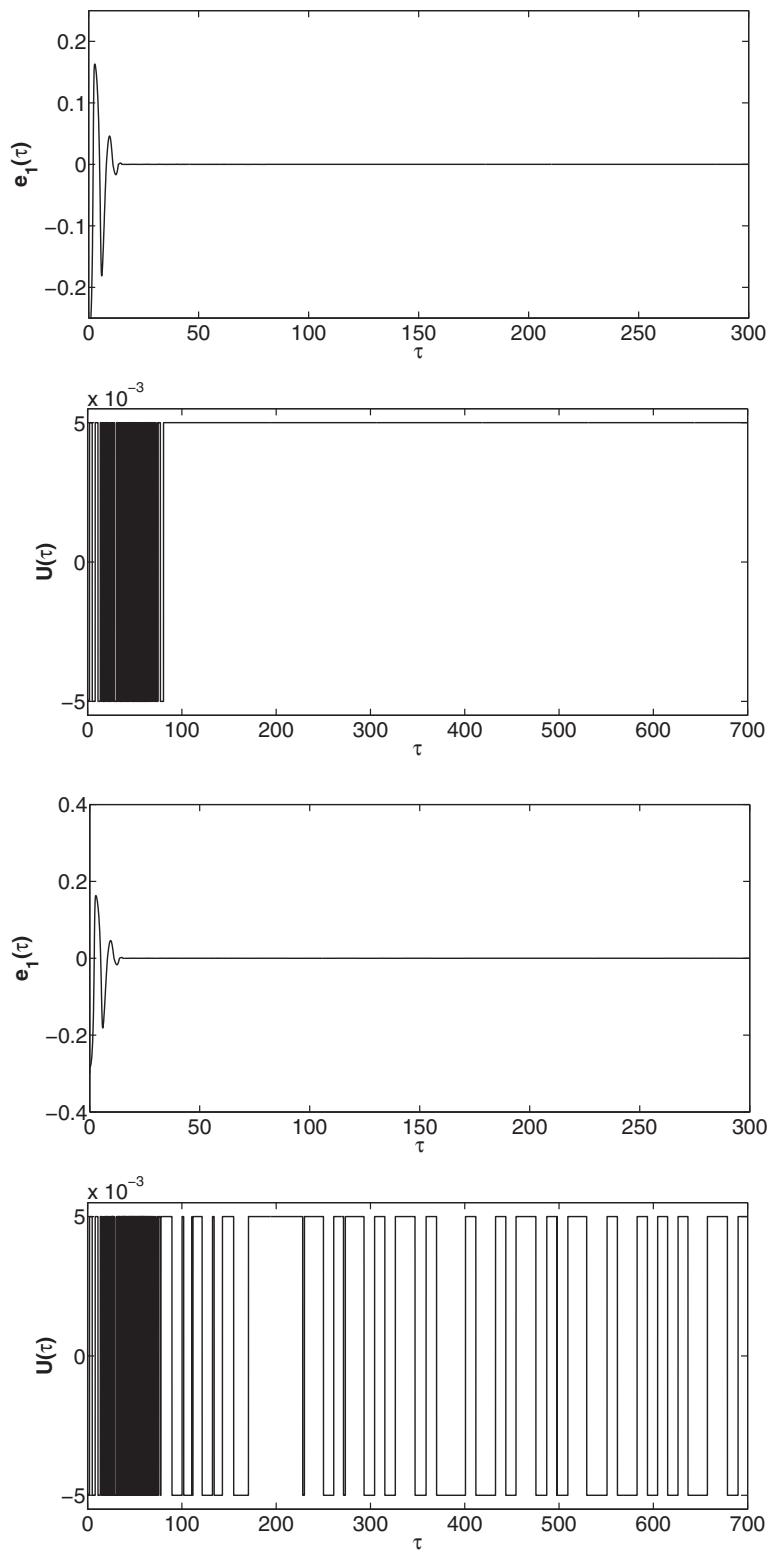

FIG. 9. Behaviors of $e_{1}(\tau)$ and $u(\tau)$. (a and b) $p=0.0017$. (c and d) $p=0.02$.

of each drive system and response system. Thus, the master and slave systems become, respectively,

$$
\begin{aligned}
& \dot{x}_{1}(\tau)=\alpha\left\{x_{2}-x_{1}-\operatorname{rf}\left[x_{1}(\tau-\theta)\right]\right\} \\
& \dot{x}_{2}(\tau)=\beta\left(x_{1}-x_{2}+r x_{3}\right) \\
& \dot{x}_{3}(\tau)=\gamma\left(E-x_{2}\right)
\end{aligned}
$$

and

$$
\begin{aligned}
\dot{y}_{1}= & \alpha\left\{y_{2}-y_{1}-r f\left[y_{1}(\tau-\theta)\right]\right\} \\
& -\zeta k \operatorname{sign}\left(y_{1}-x_{1}\right)-\zeta u(\tau) \\
\dot{y}_{2}= & \beta\left[y_{1}-y_{2}+r y_{3}-2\left(y_{1}-x_{1}\right)\right] \\
\dot{y}_{3}= & \gamma\left[E-y_{2}\right],
\end{aligned}
$$

where $\theta$ is the time delay.
For such a case, we define the functions $\phi_{1}\left(x_{1}(\tau-\right.$ $\left.\theta), y_{1}(\tau-\theta)\right)=r\left\{f\left[y_{1}(\tau-\theta)\right]-f\left[x_{1}(\tau-\theta)\right]\right\}$ and $e_{1}(\tau-$ $\theta)=y_{1}(\tau-\theta)-x_{1}(\tau-\theta)$. Thus the error state is given by the following set of equations:

$$
\begin{aligned}
\dot{e}_{1}= & \alpha\left[e_{2}-e_{1}-\phi_{1}\left(x_{1}(\tau-\theta), y_{1}(\tau-\theta)\right)\right] \\
& -\zeta k \operatorname{sign}\left(e_{1}\right)-\zeta u(\tau) \\
\dot{e}_{2}= & \beta\left[-e_{1}-e_{2}+r e_{3}\right] \\
\dot{e}_{3}= & -\gamma e_{2} .
\end{aligned}
$$

From here, using the same controller as in the previous section, we choose the following Krasovskii-Lyapunov function candidate $[28,31]$ :

$$
\begin{aligned}
V= & \frac{1}{2}\left(\frac{e_{1}^{2}}{\alpha}+\frac{e_{2}^{2}}{\beta}+\frac{r e_{3}^{2}}{\gamma}\right)+|u(\tau)| \\
& +\eta \int_{-\theta}^{0} e_{1}^{2}(\tau+s) d s,
\end{aligned}
$$

where $\eta$ is a positive constant to be determined.

The time derivative along the trajectories of the system Eq. (16) yields

$$
\begin{aligned}
\dot{V}= & -(1-\eta) e_{1}^{2}-e_{2}^{2}-\phi_{1}(\tau-\theta) e_{1}-\frac{\zeta k}{\alpha}\left|e_{1}\right| \\
& -\frac{\zeta u(\tau)}{\alpha} e_{1}+\operatorname{sign}(u) \dot{u}(\tau)-\eta e_{1}^{2}(\tau-\theta), \\
\dot{V} \leqslant & -(1-\eta) e_{1}^{2}-e_{2}^{2}+\left|\phi_{1}(\tau-\theta)\right|\left|e_{1}\right|-\frac{\zeta k}{\alpha}\left|e_{1}\right| \\
& -\frac{\zeta u(\tau)}{\alpha} e_{1}+\operatorname{sign}(u) \dot{u}(\tau)-\eta e_{1}^{2}(\tau-\theta) .
\end{aligned}
$$

Let us assume that $\left|\phi_{1}(\tau-\theta)\right| \leqslant \chi_{1}\left|e_{1}(\tau-\theta)\right|$, where $\chi_{1}$ is a positive constant. It follows from here that

$$
\begin{aligned}
\dot{V} \leqslant & -e_{1}^{2}-e_{2}^{2}+\chi_{1}\left|e_{1}(\tau-\theta)\right|\left|e_{1}\right|-\frac{\zeta k}{\alpha}\left|e_{1}\right|-\frac{\zeta u(\tau)}{\alpha} e_{1} \\
& +\operatorname{sign}(u) \dot{u}(\tau)+\eta e_{1}^{2}-\eta e_{1}^{2}(\tau-\theta), \\
\dot{V} \leqslant & -\left(1-\frac{\chi_{1}}{2}-\eta\right) e_{1}^{2}-e_{2}^{2}+\left(\frac{\chi_{1}}{2}-\eta\right) e_{1}^{2}(\tau-\theta) \\
& -\frac{\zeta k}{\alpha}\left|e_{1}\right|-\frac{\zeta u(\tau)}{\alpha} e_{1}+\operatorname{sign}(u) \dot{u}(\tau) .
\end{aligned}
$$

Let $\eta=\frac{\chi_{1}}{2}, \chi_{1}<1$, and $\zeta=\alpha$. It follows that

$$
\dot{V} \leqslant-k\left|e_{1}\right|-u(\tau) e_{1}+\operatorname{sign}(u) \dot{u}(\tau) .
$$

Thus, using the controller

$$
\dot{u}=\operatorname{sign}(u)\left(k\left|e_{1}\right|+u e_{1}-p\right),
$$

it follows that

$$
\dot{V} \leqslant-p
$$

Hence, global finite-time stability is achieved [15]. For any time $\tau$ contained in the interval $0<\tau<\theta$ both the drive and response system do not oscillate at the considered regime. Therefore, to determine the theoretical finite settling time, we integrate Eq. (22) from $\theta$ to $\tau_{s}$ and we obtain

$$
V\left(\tau_{s}\right)-V(\theta) \leqslant-p\left(\tau_{s}-\theta\right) .
$$


Using the fact that $V\left(\tau_{s}\right) \rightarrow 0$ when $\tau \rightarrow \tau_{s}, V>0 \forall \tau$ and, taking into account Eq. (22), we can see that the Lyapunov function $V$ is a monotonous and decreasing function. It follows that

$$
\begin{aligned}
\tau_{s}= & \theta+\frac{1}{2 p}\left(\frac{1}{\alpha} e_{1}^{2}(\theta)+\frac{1}{\beta} e_{2}^{2}(\theta)+\frac{R}{\gamma} e_{3}^{2}(\theta)\right)+\frac{|u(\theta)|}{p} \\
& +\eta \int_{-\theta}^{0} \varepsilon_{1}^{2}(\theta+s) d s \\
\tau_{s} \geqslant \theta & +\frac{1}{2 p}\left(\frac{1}{\alpha} e_{1}^{2}(\theta)+\frac{1}{\beta} e_{2}^{2}(\theta)+\frac{R}{\gamma} e_{3}^{2}(\theta)\right)+\frac{|u(\theta)|}{p} .
\end{aligned}
$$

Equation (24) gives the maximum settling time for synchronization. Hence, the finite-time synchronization is reached when the numerical settling time $\tau_{s \mathrm{NU}}$ satisfies the relation $\tau_{s \mathrm{NU}}<\tau_{s \mathrm{TH}}$, where $\tau_{s \mathrm{TH}}=\theta+\frac{1}{2 p}\left(\frac{1}{\alpha} e_{1}^{2}(\theta)+\frac{1}{\beta} e_{2}^{2}(\theta)+\right.$ $\left.\frac{R}{\gamma} e_{3}^{2}(\theta)\right)+\frac{|u(\theta)|}{p}$.

\section{Numerical results}

In this section we investigate the finite-time synchronization of two delayed tunnel-diode-based chaotic systems basing ourselves on the obtained numerical results of the established theory in the previous subsection. The integration time step taken was $10^{-4}$. Figure 10 helps to confirm the synchronization behavior of both the delayed master system [Eq. (14)] and the slave system [Eq. (15)] when $p=0.001$ and $k=0.005$. With the selected values of parameters $p$ and $k$, the theoretical settling time result is $\tau_{s \mathrm{TH}} \simeq 127.5448$, while the numerical settling time corresponding to the condition given before is $\tau_{s \mathrm{NU}} \simeq 99.4$.

When varying the time lag $\theta$, just the theoretical settling time is modified when synchronization occurs and the numerical finite time is determined as $\tau_{s \mathrm{NU}} \simeq 99.4$.

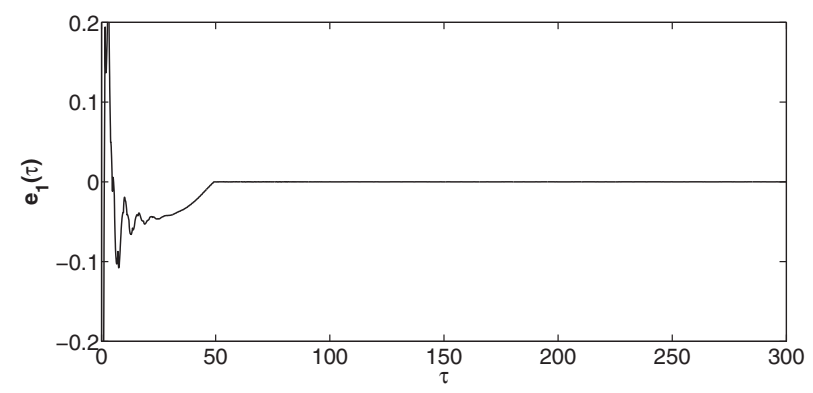

(a)

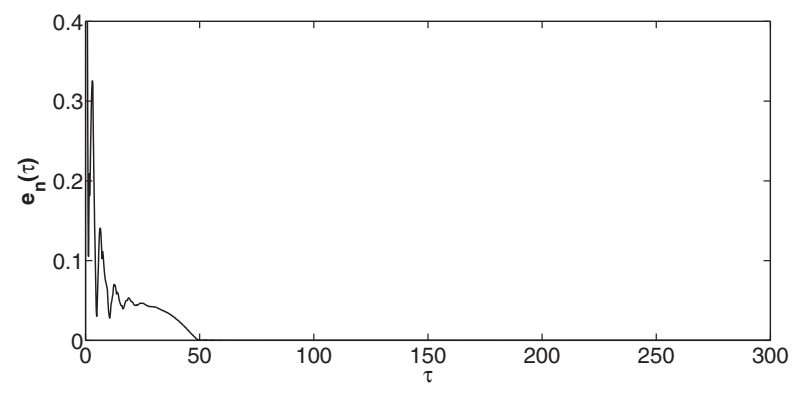

\section{B. Multidelayed systems}

\section{Synchronization analysis}

In this section we consider that there exist two delays: $\theta_{1}$ represents the time lag taken for the introduction of the nonlinearity, and $\theta_{2}$ represents the time delay between the master state and the slave state. Thus, the systems become

$$
\begin{aligned}
\dot{x}_{1}\left(\tau-\theta_{2}\right)= & \alpha\left\{x_{2}\left(\tau-\theta_{2}\right)-x_{1}\left(\tau-\theta_{2}\right)\right. \\
& \left.-r f\left[x_{1}\left(\tau-\theta_{1}-\theta_{2}\right)\right]\right\} \\
\dot{x}_{2}\left(\tau-\theta_{2}\right)= & \beta\left[x_{1}\left(\tau-\theta_{2}\right)-x_{2}\left(\tau-\theta_{2}\right)\right. \\
& \left.+r x_{3}\left(\tau-\theta_{2}\right)\right] \\
\dot{x}_{3}\left(\tau-\theta_{2}\right)= & \gamma\left[E-x_{2}\left(\tau-\theta_{2}\right)\right]
\end{aligned}
$$

and

$$
\begin{aligned}
\dot{y}_{1}(\tau)= & \alpha\left\{y_{2}(\tau)-y_{1}(\tau)-r f\left[y_{1}\left(\tau-\theta_{1}\right)\right]\right\} \\
& -\zeta k \operatorname{sign}\left[y_{1}(\tau)-x_{1}\left(\tau-\theta_{2}\right)\right]-\zeta u(\tau) \\
\dot{y}_{2}(\tau)= & \beta\left\{y_{1}(\tau)-y_{2}(\tau)+r y_{3}\right. \\
& \left.-2\left[y_{1}(\tau)-x_{1}\left(\tau-\theta_{2}\right)\right]\right\} \\
\dot{y}_{3}(\tau)= & \gamma\left[E-y_{2}(\tau)\right] .
\end{aligned}
$$

In this case, we define the functions $\phi_{2}\left(\tau, \theta_{1}, \theta_{2}\right)=$ $r\left\{f\left[y_{1}\left(\tau-\theta_{1}\right)\right]-f\left[x_{1}\left(\tau-\theta_{1}-\theta_{2}\right)\right]\right\} \quad$ and $e_{1}\left(\tau-\theta_{1}-\right.$ $\left.\theta_{2}\right)=y_{1}\left(\tau-\theta_{1}\right)-x_{1}\left(\tau-\theta_{1}-\theta_{2}\right)$. Thus the error state is given by the following set of equations:

$$
\begin{aligned}
\dot{e}_{1}= & \alpha\left[e_{2}-e_{1}-\phi_{2}\left(\tau, \theta_{1}, \theta_{2}\right)\right] \\
& -\zeta k \operatorname{sign}\left(e_{1}\right)-\zeta u(\tau) \\
\dot{e}_{2}= & \beta\left[-e_{1}-e_{2}+r e_{3}\right] \\
\dot{e}_{3}= & -\gamma e_{2} .
\end{aligned}
$$

Let us now select the Krasovskii-Lyapunov function as [28,31]

$$
\begin{aligned}
V= & \frac{1}{2}\left(\frac{e_{1}^{2}}{\alpha}+\frac{e_{2}^{2}}{\beta}+\frac{r e_{3}^{2}}{\gamma}\right)+|u(\tau)| \\
& +\lambda \int_{-\theta_{1}}^{0} e_{1}^{2}\left(\tau-\theta_{2}+s\right) d s,
\end{aligned}
$$

where $\lambda$ is a positive constant to be determined.

The time derivative along the trajectories of the system Eq. (27) yields

$$
\begin{aligned}
\dot{V}= & -(1-\lambda) e_{1}^{2}-e_{2}^{2}-\phi_{2}\left(\tau, \theta_{1}, \theta_{2}\right) e_{1}-\frac{\zeta k}{\alpha}\left|e_{1}\right| \\
& -\frac{\zeta u(\tau)}{\alpha} e_{1}+\operatorname{sign}(u) \dot{u}(\tau)-\lambda e_{1}^{2}\left(\tau-\theta_{1}-\theta_{2}\right), \\
\dot{V} \leqslant & -(1-\lambda) e_{1}^{2}-e_{2}^{2}+\left|\phi_{2}\left(\tau, \theta_{1}, \theta_{2}\right)\right|\left|e_{1}\right|-\frac{\zeta k}{\alpha}\left|e_{1}\right| \\
& -\frac{\zeta u(\tau)}{\alpha} e_{1}+\operatorname{sign}(u) \dot{u}(\tau)-\lambda e_{1}^{2}\left(\tau-\theta_{1}-\theta_{2}\right) .
\end{aligned}
$$

Let us assume that $\left|\phi_{2}\left(\tau, \theta_{1}, \theta_{2}\right)\right| \leqslant \chi_{2}\left|e_{1}\left(\tau-\theta_{1}-\theta_{2}\right)\right|$, where $\chi_{2}$ is a positive constant. It follows from $\sqrt{e_{1}^{2}(\tau)+e_{2}^{2}(\tau)+e_{3}^{2}(\tau)}$ 
here that

$$
\begin{aligned}
\dot{V} \leqslant & -e_{1}^{2}-e_{2}^{2}+\chi_{2}\left|e_{1}\left(\tau-\theta_{1}-\theta_{2}\right)\right|\left|e_{1}\right|-\frac{\zeta k}{\alpha}\left|e_{1}\right| \\
& -\frac{\zeta u(\tau)}{\alpha} e_{1}+\operatorname{sign}(u) \dot{u}(\tau)+\lambda e_{1}^{2}-\lambda e_{1}^{2}\left(\tau-\theta_{1}-\theta_{2}\right), \\
\dot{V} \leqslant & -\left(1-\frac{\chi_{2}}{2}-\lambda\right) e_{1}^{2}+\left(\frac{\chi_{2}}{2}-\lambda\right) e_{1}^{2}\left(\tau-\theta_{1}-\theta_{2}\right) \\
& -\frac{\zeta k}{\alpha}\left|e_{1}\right|-e_{2}^{2}-\frac{\zeta u(\tau)}{\alpha} e_{1}+\operatorname{sign}(u) \dot{u}(\tau) .
\end{aligned}
$$

Let $\lambda=\frac{\chi_{2}}{2}, \chi_{2}<1$, and $\zeta=\alpha$. It follows that

$$
\dot{V} \leqslant-k\left|e_{1}\right|-u(\tau) e_{1}+\operatorname{sign}(u) \dot{u}(\tau) .
$$

Thus, using the controller

$$
\dot{u}=\operatorname{sign}(u)\left(k\left|e_{1}\right|+u e_{1}-p\right),
$$

it follows that

$$
\dot{V} \leqslant-p .
$$

Hence, the global finite-time stability is achieved [15]. When $0<\tau<\theta_{1}+\theta_{2}$ the system cannot oscillate at the considered regime. In order to determine the theoretical finite settling time, we integrate Eq. (33) from $\theta_{1}+\theta_{2}$ to $\tau_{s}$. Thus, the finite settling time is given by

$$
\begin{aligned}
\tau_{s}= & \frac{1}{2 p}\left(\frac{1}{\alpha} e_{1}^{2}\left(\theta_{1}+\theta_{2}\right)+\frac{1}{\beta} e_{2}^{2}\left(\theta_{1}+\theta_{2}\right)+\frac{R}{\gamma} e_{3}^{2}\left(\theta_{1}+\theta_{2}\right)\right) \\
& +\frac{\left|u\left(\theta_{1}+\theta_{2}\right)\right|}{p}+\lambda \int_{-\theta_{1}+\theta_{2}}^{0} \epsilon_{1}^{2}\left(\theta_{1}+\theta_{2}+s\right) d s+\theta_{1}+\theta_{2}, \\
\tau_{s} \geqslant & \frac{1}{2 p}\left(\frac{1}{\alpha} e_{1}^{2}\left(\theta_{1}+\theta_{2}\right)+\frac{1}{\beta} e_{2}^{2}\left(\theta_{1}+\theta_{2}\right)+\frac{R}{\gamma} e_{3}^{2}\left(\theta_{1}+\theta_{2}\right)\right) \\
& +\frac{\left|u\left(\theta_{1}+\theta_{2}\right)\right|}{p}+\theta_{1}+\theta_{2} .
\end{aligned}
$$

At this moment, as in the previous section, the finite-time synchronization is reached when the numerical settling time $\tau_{s \mathrm{NU}}$ satisfies the relation $\tau_{s \mathrm{NU}}<\tau_{s \mathrm{TH}}$, where

$$
\begin{aligned}
\tau_{s \mathrm{TH}}= & \frac{1}{2 p}\left(\frac{1}{\alpha} e_{1}^{2}\left(\theta_{1}+\theta_{2}\right)+\frac{1}{\beta} e_{2}^{2}\left(\theta_{1}+\theta_{2}\right)+\frac{R}{\gamma} e_{3}^{2}\left(\theta_{1}+\theta_{2}\right)\right) \\
& +\frac{\left|u\left(\theta_{1}+\theta_{2}\right)\right|}{p}+\theta_{1}+\theta_{2} .
\end{aligned}
$$

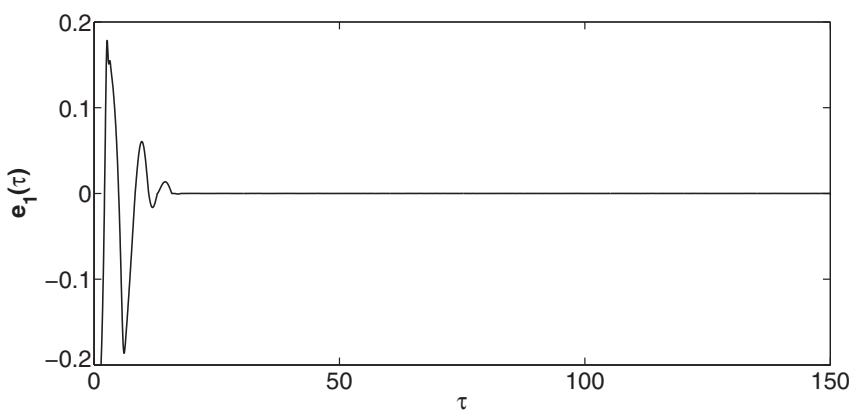

FIG. 11. Time history of $e_{1}(\tau)$.
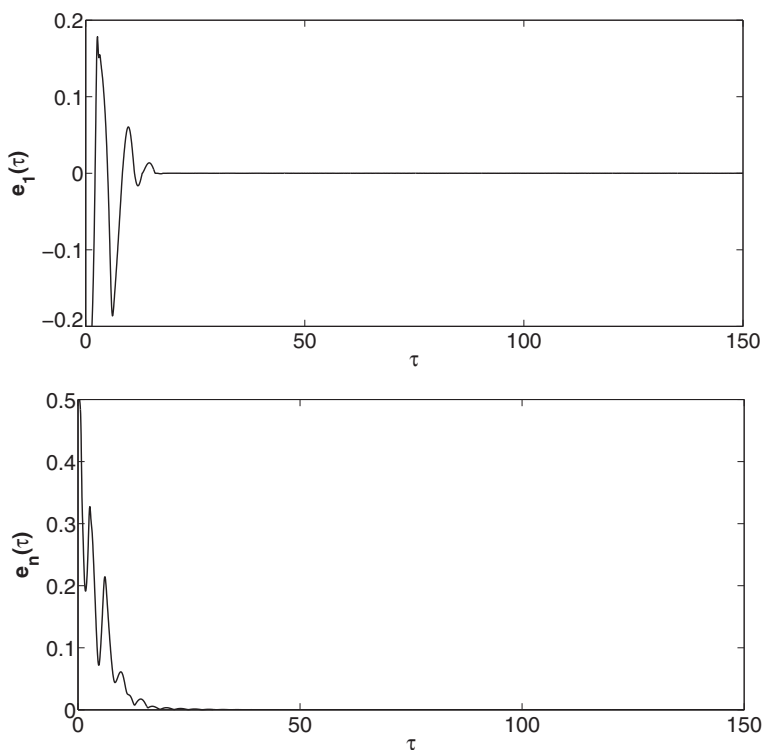

FIG. 12. Time history of (a) $e_{1}(\tau)$ and (b) $e_{n}(\tau)$ when $\theta_{2}=0.0003$.

\section{Numerical results}

From our investigations, it comes out that the finite-time synchronization is hardly reached when we consider that both dynamics of the drive system and response system are subjected to delay $\theta_{2}$. The integration time step used in this part was $10^{-4}$. The graphs in Fig. 11 show that the goal can be achieved only if $\theta_{2}$ is relatively small. For the values $k=0.005$, $p=0.001, \theta_{1}=0.1$, and $\theta_{2}=0.0003$, one has the following behavior.

Considering the behavior of the synchronization error $e_{1}(\tau)$ and $e_{n}(\tau)$ (defined in Fig. 10), one can observe the destruction of the synchronization when we increase the value of $\theta_{2}$ (Figs. 12-14).

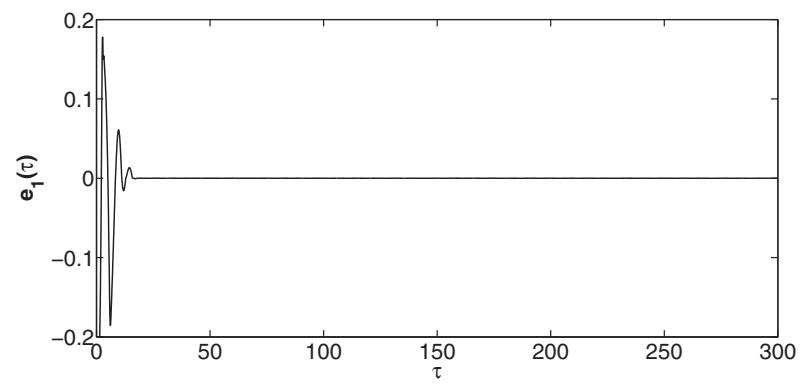

(a)

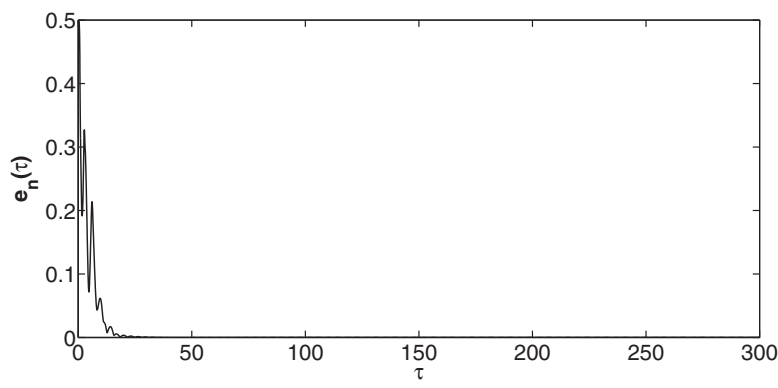




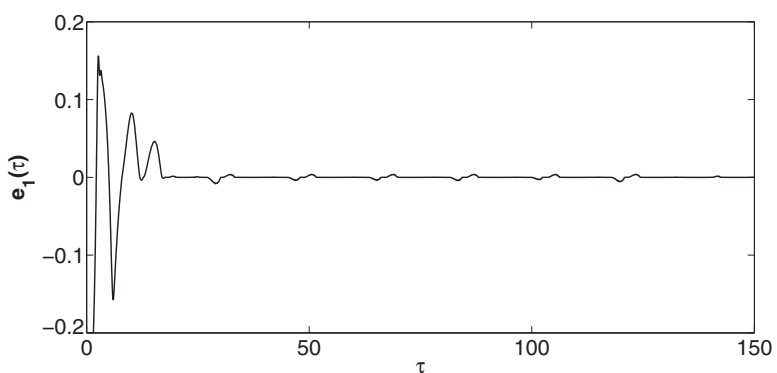

(a)

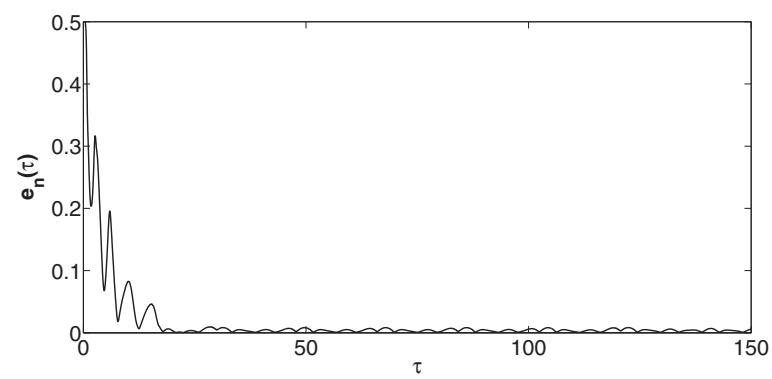

(b)

FIG. 14. Time history of (a) $e_{1}(\tau)$ and (b) $e_{n}(\tau)$ when $\theta_{2}=0.08$.

\section{CONCLUSIONS}

The target of this paper was to investigate the possibility to achieve synchronization in finite time of two tunnel-diodebased chaotic oscillators. We have considered the case of chaotic systems without and with delay (internal delay and multiple delay). The controller was built basing ourselves on the absolute stability theory [28] and on the Krasovskii-

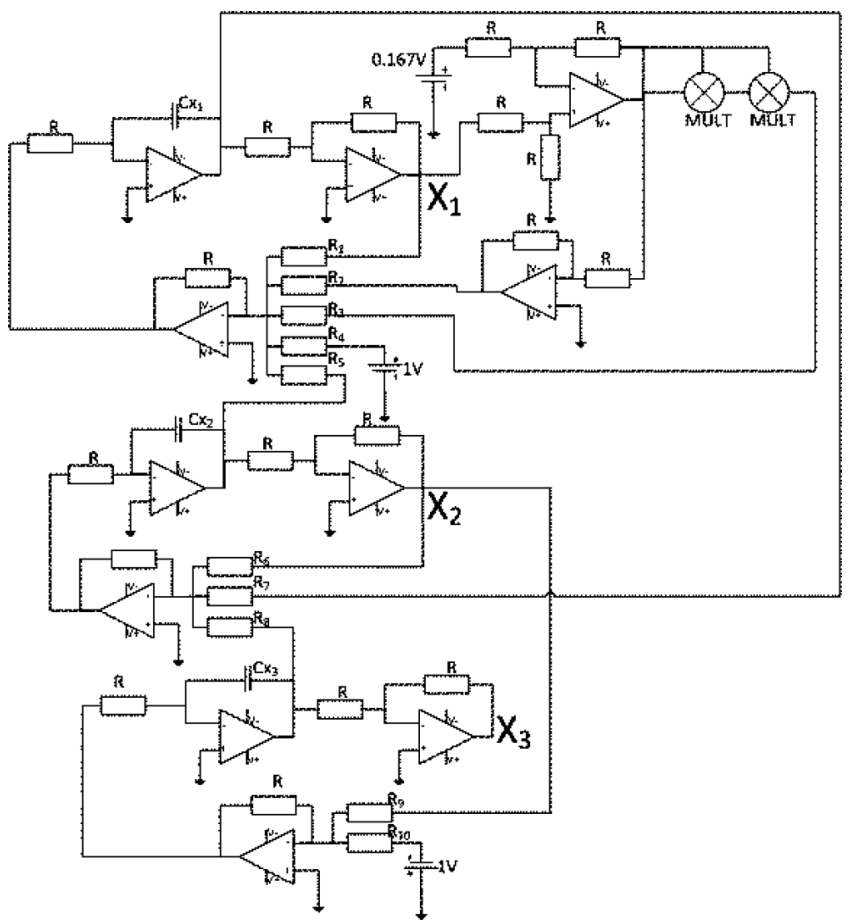

FIG. 15. Circuit diagram of the analog Tunnel Diode based Oscillator system.

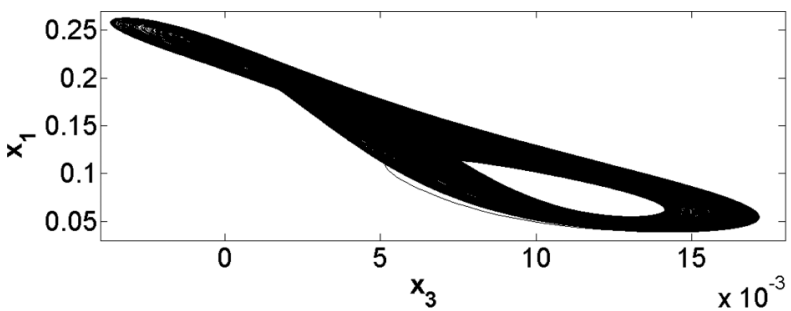

(a)

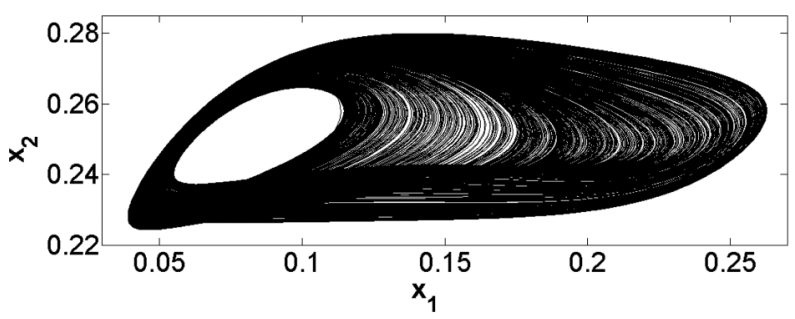

(b)

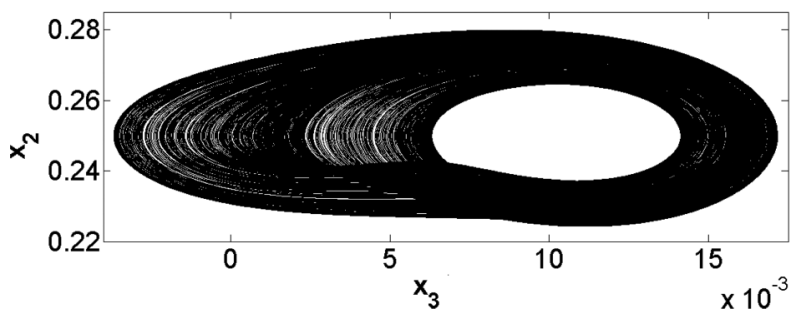

FIG. 16. Chaotic attractor from the circuit in Fig. 15.

Lyapunov stability theory [31]. Later, the expression of the settling finite time was investigated in all considered cases. Numerical simulations were performed and given to confirm our theoretical analysis. We observe that the finite-time

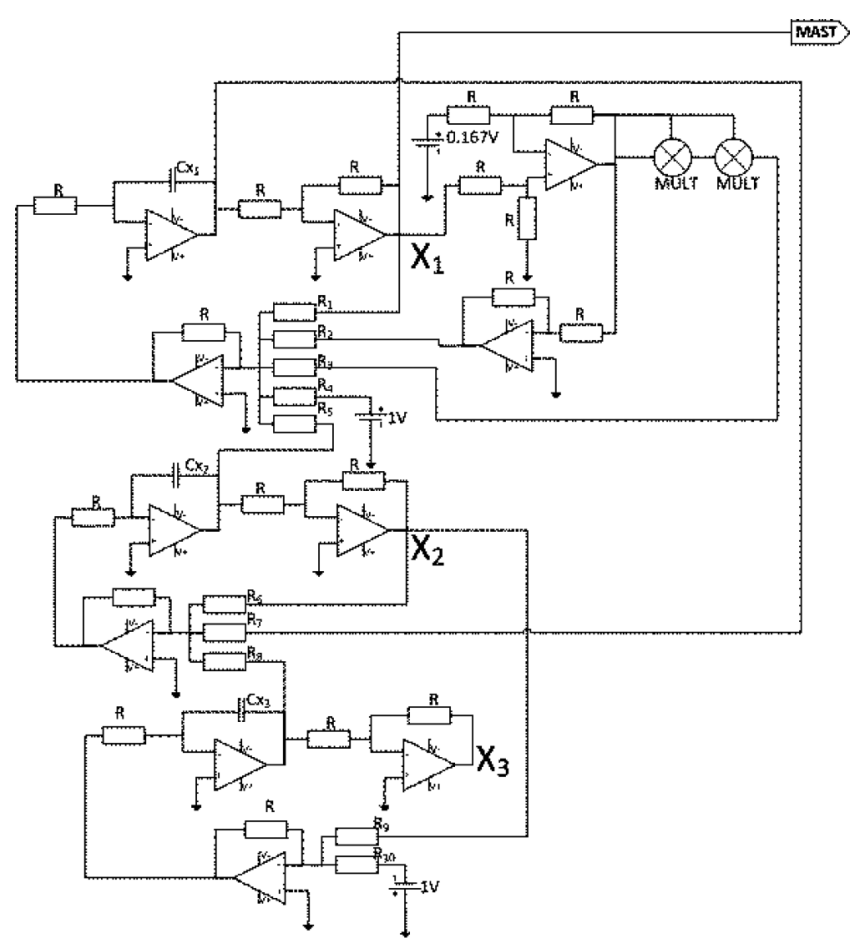

FIG. 17. Circuit diagram of the drive system. 


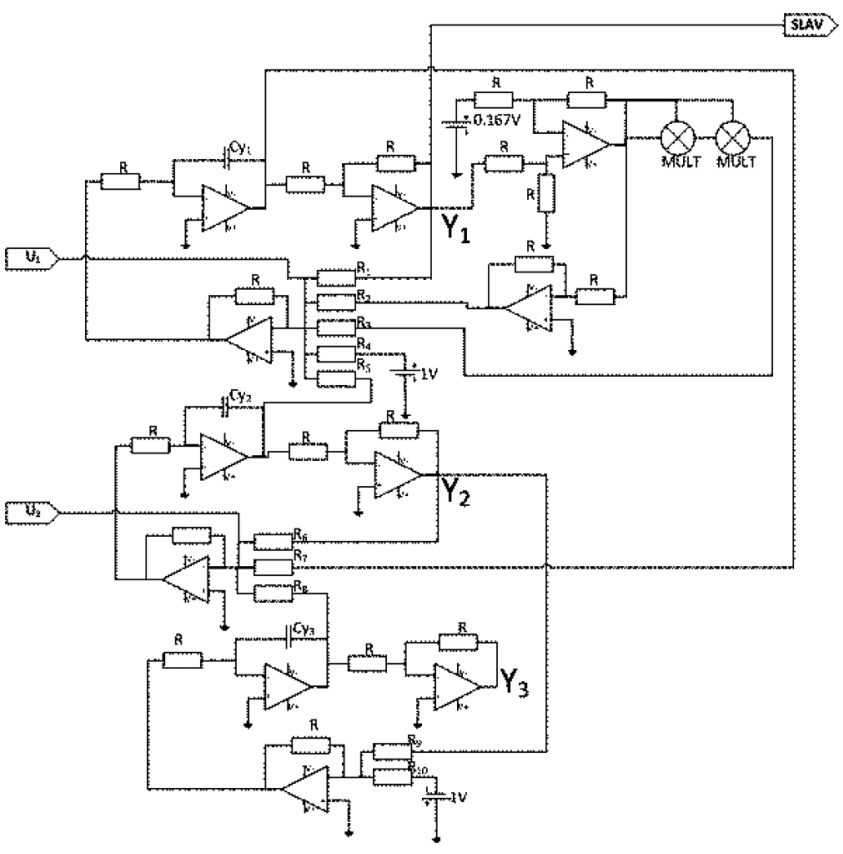

FIG. 18. Circuit diagram of the response system.

synchronization is reached when some conditions, which are given and proven by numerical results, are filled.

\section{APPENDIX: CIRCUIT DESIGN AND SIMULATIONS}

Here we investigate the electrical circuit of the system. First we build the analog computer of the chaotic oscillator, which is shown in Fig. 15. The use of the analog computer here is helpful because we can modify the frequency of the oscillator and thus facilitate the design of the controller.

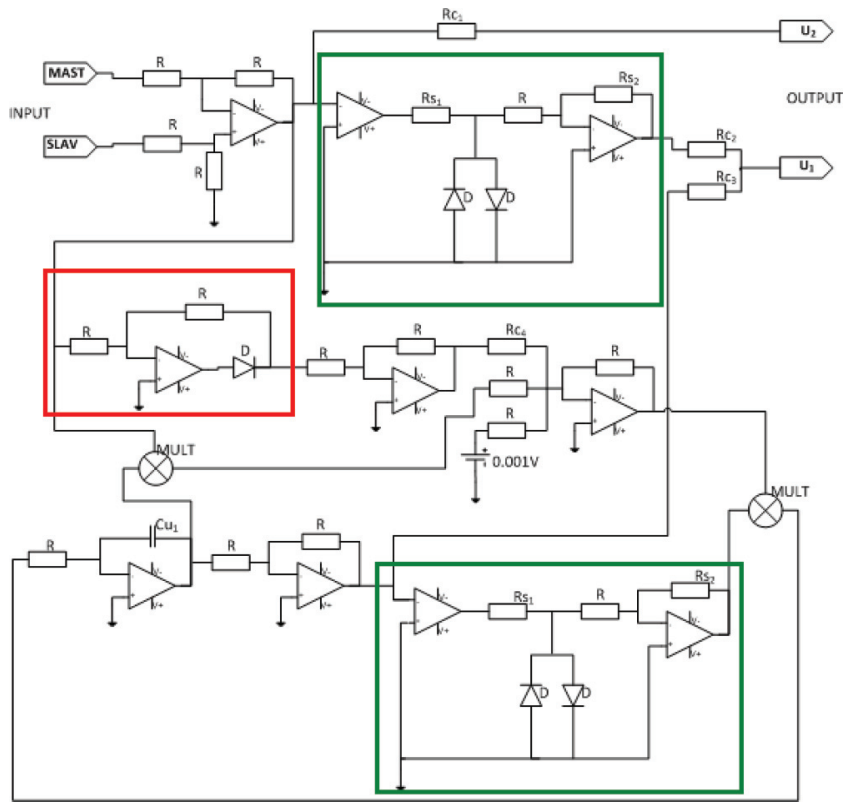

FIG. 19. (Color online) Circuit diagram of the controller.
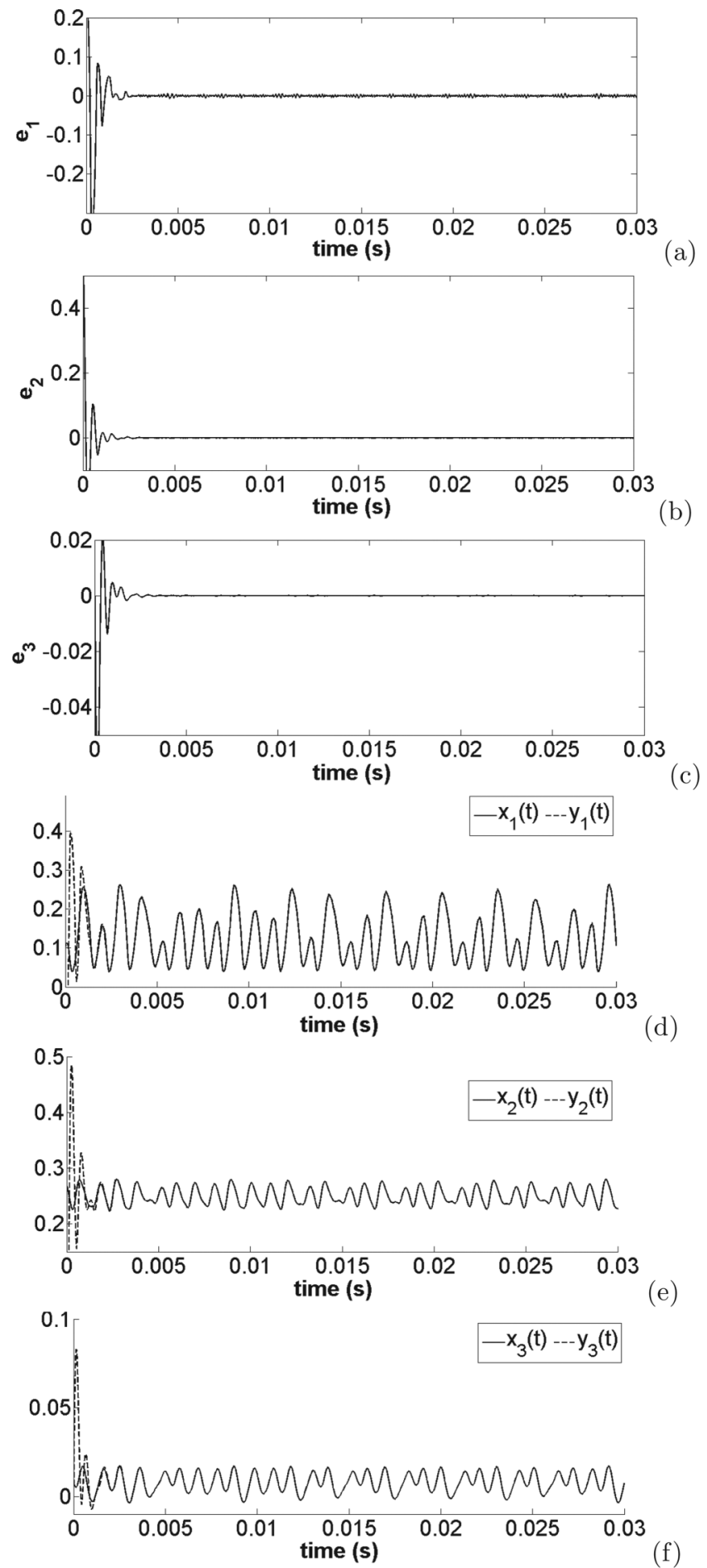

FIG. 20. Time histories of $e_{i}(t), i=1,2,3$ and of the systems variables.

This circuit is obtained through the following relations: $\alpha=\frac{1}{\chi R_{1} C_{x_{1}}}=\frac{1}{\chi R_{5} C_{x_{1}}}, \alpha r a_{1}=\frac{1}{\chi R_{3} C_{x_{1}}}, \alpha r a_{2}=\frac{1}{\chi R_{2} C_{x_{1}}}, \alpha r a_{3}=$ $\frac{1}{\chi R_{4} C_{x_{1}}}, \beta=\frac{1}{\chi R_{7} C_{x_{2}}}=\frac{1}{\chi R_{6} C_{x_{2}}}, \beta r=\frac{1}{\chi R_{8} C_{x_{2}}}, \gamma=\frac{1}{\chi R_{9} C_{x_{3}}}$, and $\gamma E=\frac{1}{\chi R_{10} C_{x_{3}}}$, where $\chi$ is a parameter chosen as $\chi=$ $10^{4}$. Thus, considering that $R=10 \mathrm{k} \Omega$ and all capacitors are $C=10 n F$, we obtain the following component values: 
$R_{1}=R_{5}=3988.095238 \Omega, \quad R_{3}=188.2189546 \Omega, \quad R_{2}=$ $3.48 \mathrm{k} \Omega, \quad R_{4}=46261.31262 \Omega, \quad R_{6}=R_{7}=33.5 \mathrm{k} \Omega, \quad R_{8}=$ $2093.75 \Omega, R_{9}=47761.19403 \Omega$, and $R_{10}=191044.7761 \Omega$. The chaotic behavior of the above circuit is given by the graphs in Fig. 16.

Later, the drive (Fig. 17) and response (Fig. 18) systems are designed as follows. The controller (Fig. 19) is designed using the following parameters: $2 \beta=\frac{1}{\chi R_{C_{1}} C_{y_{2}}}, \zeta k=\frac{1}{\chi R_{C_{2}} C_{y_{1}}}$, $\zeta=\frac{1}{\chi R_{C_{3}} C_{y_{1}}}, k=\frac{1}{\chi R_{C_{4}} C_{u_{1}}}, 1=\frac{1}{\chi R C_{u_{1}}}$, and $p=\frac{0.001}{\chi R C_{u_{1}}}$, with which we obtain the component values: $R_{C_{1}}=16.75 \mathrm{k} \Omega$, $R_{C_{2}}=797619.0476 \Omega, R_{C_{3}}=3988.095238 \Omega, R_{C_{4}}=2 M \Omega$, $R_{S_{1}}=1 \mathrm{k} \Omega$, and $R_{S_{2}}=13.508 \mathrm{k} \Omega$. The part of the controller circuit in the red box, according to J. C. Sprott [29], simulates the absolute value function, while the ones in the green domains simulate the sign functions [30]. The results obtained from these circuits are given in Fig. 20.
[1] B. Blasius, A. Huppert, and L. Stone, Nature (London) 399, 354 (1999).

[2] D. W. Graham, C. W. Knapp, E. S. Van Vleck, K. Bloor, Teresa B. Lane, and C. E. Graham, Int. Soc. Microb. Ecol. J. 1, 385 (2007).

[3] P. Louodop, H. Fotsin, and S. Bowong, Phys. Scr. 85, 025002 (2012).

[4] S. Bowong and J. Kurths, Phys. Lett. A 374, 4496 (2010).

[5] A. Casadevall, F. C. Fang, and L.-A. Pirofski, PLoS Pathog. 7, e1002136 (2011).

[6] P. Philippe, Hum. Biol. 65, 525 (1993).

[7] D. J. D. Earn, P. Rohanian, and B. Y. Grenfell, Proc. R. Soc. London B 265, 7 (1998).

[8] E. Beninca, J. Huisman, R. Heerkloss, K. D. Johnk, P. Branco, E. H. van Nes, M. Scheffer, and S. P. Ellner, Nature (London) 451, 822 (2008).

[9] C. Cruz-Hernandez, Nonlinear Dynamics and Systems Theory 4, 1 (2004)

[10] S. Bowong and J. J. Tewa, Math. Comput. Model. 48, 1826 (2008).

[11] M. Feki, Chaos Solitons Fractals 18, 141 (2003).

[12] W. Yang, X. Xia, Y. Dong, and S. Zheng, Computer and Information Science 3, 174 (2010).

[13] Z. Gui, X. Wu, and Q. Lin, in Proceedings of the 2009 International Workshop on Chaos-Fractals Theories and Applications (IEEE Computer Society, Washington, DC, 2009), p. 16.

[14] M. P. Lazarevi and D. Lj. Debeljkovi, Asian J. Control 7, 440 (2005).

[15] L. A. Zuppa, C. C. Hérnandez, and A. Y. A. Bustos, http://www. wseas.us/e-library/conferences/mexico2002/papers/249.pdf.

[16] M. Lakshmanan and D. V. Senthilkumar, Dynamics of Nonlinear Time-Delay Systems (Springer-Verlag, New York, 2010).
[17] E. R. Brown, J. R. Soderstrom, C. D. Parker, L. J. Mahoney, K. M. Molvar, and T. C. McGill, Appl. Phys. Lett. 58, 2291 (1991).

[18] S. Suzuki, A. Teranishi, K. Hinata, M. Asada, H. Sugiyama, and H. Yokoyama, Appl. Phys. Express. 2, 054501 (2009).

[19] L. Wang, Ph.D. thesis, Glasgow University, 2011, http://theses.gla.ac.uk/3423/.

[20] H. P. Ren, M. S. Baptista, and C. Grebogi, Phys. Rev. Lett. 110, 184101 (2013).

[21] B. S. Dmitriev, A. E. Hramov, A. A. Koronovskii, A. V. Starodubov, D. I. Trubetskov, and Y. D. Zharkov, Phys. Rev. Lett. 102, 074101 (2009).

[22] A. A. Koronovskii, O. I. Moskalenko, and A. E. Hramov, Phys. Usp. 52, 1213 (2009).

[23] Y. Liu, Nonlinear Dynamics 67, 89 (2012)

[24] H. Wang, Z. Han, Q. Xie, and W. Zhang, Nonlinear Analysis: Real World Applications 10, 2842 (2009).

[25] A. S. Pikovskii and M. I. Rabinovich, Sov. Phys. Dokl. 23, 183 (1978).

[26] A. Yu. Markov, A. L. Fradkov, and G. S. Simin, in Proceedings of the 35th Conference on Decision and Control, Kobe, Japan (IEEE, http://www.ipme.ru/ipme/labs/ccs/alf/mfs_cdc96.pdf, 1996), pp. 2177-2182.

[27] M. T. Yassen, Appl. Math. Comput. 135, 113 (2003).

[28] X. Liao and P. Yu, Absolute Stability of Nonlinear Control Systems (Springer, New York, 2008).

[29] J. C. Sprott, Phys. Lett. A 266, 19 (2000).

[30] J. Lin, C. Huang, T. Liao, and J. Yan, Digit. Signal Process. 20, 229 (2010).

[31] D. V. Senthilkumar, J. Kurths, and M. Lakshmanan, Phys. Rev. E 79, 066208 (2009) 